\title{
Gesundheit fördert Integration fördert Gesundheit
}

Viele Jahrzehnte hat die Politik die Migrationswirklichkeit in geradezu ritualisierter Leerformeldiskussion verdrängt. Zwischenzeitlich weist ein Fünftel der deutschen Bevölkerung einen Migrationshintergrund auf. Deutschland ist damit in Europa eines der größten Zuwanderungsländer. Der vorliegende Beitrag beschäftigt sich mit der Frage, welche Voraussetzungen geschaffen werden müssen, damit die Gesundheit integrationsfördernd und die Integration wiederum gesundheitsfördernd wirken kann.

\section{Sigrid Pettrup}

\section{Migrationshintergrund}

Von den knapp 82 Millionen der in Deutschland lebenden Menschen kommen 15,3 Millionen aus dem Ausland bzw. haben einen so genannten Migrationshintergrund. Dies entspricht 19\% der gesamten Bevölkerung ${ }^{1}$. $10 \%$ haben einen deutschen Pass, $9 \%$ einen ausländischen. Der Anteil der Personen mit Migrationshintergrund ist demnach fast doppelt so groß wie der Anteil der Personen, die als Ausländer erfasst sind.

Mit den Ergebnissen des Mikrozensus'2005 wird erstmals die tatsächliche Bevölkerungsstruktur mit Zahlen belegbar: ein Fünftel der bundesdeutschen Bevölkerung ist ausländischer Herkunft bzw. hat Eltern oder Großeltern, die aus dem Ausland stammen, ist also auf die eine oder andere Weise ausländischer Herkunft. ${ }^{2}$

Als Menschen mit Migrationshintergrund gelten sowohl Zugewanderte, die das Ereignis der Zuwanderung in ihrer persönlichen Biografie erlebt haben, in Deutschland Geborene von Eltern mit Zuwanderungsgeschichte, die qua Geburt die deutsche Staatsbürgerschaft erwerben als auch eingebürgerte Ausländer und Spätaussiedler beziehungsweise deren Kinder, die schon in Deutschland geboren sind.

Die Daten bescheinigen, dass Deutschland sich zu einer multikulturellen bzw. multi-ethnischen Gesellschaft entwickelt hat. Prognosen der Demografen gehen davon aus, dass nach dieser Definition im Jahr 2010 ca. 40\% der Unter-40-Jährigen eine Zuwanderungsgeschichte - in welcher Form auch immer- aufweisen.

\section{Integrationspolitik}

Angesichts solcher Zahlen könne man „(...) durchaus von einer Zuwanderungsgesellschaft sprechen(...)", kommen-

Sigrid Pettrup, Abt. Gesundheitsförderung und Selbsthilfe, BKK Bundesverband, Essen tierte Johann Hahlen, Präsident des Statistischen Bundesamtes, die im Mikrozensus 2005 erhobenen Ergebnisse, welche erstmalig herkunftbezogene Fragen in differenzierter Form mit berücksichtigen.

Dieser Tatsache, Deutschland $=$ Zuwanderungsland, wird mit dem Zuwanderungsgesetz von 2005 politisch Rechnung getragen. Die Diskussion um Zuwanderungsfragen mag damit vorläufig einen rechtlichen Abschluss gefunden haben; im politischen Diskurs gewinnt sie indes zunehmend an Bedeutung.

Im Sommer letzten Jahres lud die Bundeskanzlerin zum Integrationsgipfel ein. Gedacht als Auftakt zum fortlaufenden Dialog, an dessen Abschluss ein „Nationaler Integrationsplan" mit präzisen Zielformulierungen, konkreten Maßnahmen und Selbstverpflichtungen als Grundlage einer nachhaltigen Integrationspolitik erarbeitet werden soll, war es Anliegen, Migrantinnen und Migranten sowie Vertreter aller politischen Ebenen und gesellschaftlichen Gruppen an einen Tisch und in Diskussion zu bringen.

Im Vorfeld des Gipfels legte das Bundeskabinett ein Positionspapier zur Politik der Integrationsbeauftragten vor, welche die Integration von zugewanderten Menschen als politische Schlüsselaufgabe identifiziert.

Integrationspolitik verfolgt das Ziel einer möglichst guten und breiten Verwirklichung von Beteiligungsmöglichkeiten in allen Lebensbereichen. Dies betrifft vor allem Bildung, Arbeit, Wirtschaft und Soziales, Recht, Kultur, Religion, Wohnen und - Gesundheit.

\section{Gesundheit als integrationspolitische Herausforderung}

Integration, soziologisch ein "Prozess der Bildung von Ganz- oder Einheiten aus Teilen", kann als gelungen dann bezeichnet werden, wenn eine Gesellschaft sich im 
Gleichgewicht befindet. Idealtypisch wird diese Gleichgewichtung dadurch angezeigt,

"(...) dass die Handelnden sich im Einklang mit ihren individuellen Bedürfnissen und mit den Erwartungen ihrer Interaktionspartner verhalten(...). was die (...) Verinnerlichung gemeinsamer Werte und Normen (...) voraussetzt ${ }^{3}$. Einem Verständnis von Integration auf der Basis kultureller Vielfalt entsprechend beinhaltet dies nicht, dass Integration zu einer völligen Assimilation der einzelnen hinzugefügten Teile führen muss.

Der aktuelle Diskurs um Integration zoomt - neben vielen anderen Handlungsfeldern - auch die Frage nach der gegenwärtigen praktischen Leistungseffektivität der gesundheitlicher Versorgungssysteme in Bezug auf die Klientel mit Migrationshintergrund näher ins Blickfeld. Und wirft damit Fragen auf: nach Mitbestimmung, Partizipation, interkultureller Öffnung und Kompetenzen, Ressourcennutzung, Emanzipation, Risiken, Chancen etc. auf beiden Seiten, der aufnehmenden wie der der zuwandernden/ zugewanderten Gesellschaft im Versorgungskontext. Wie steht es, so lässt sich fragen, um den bisherigen Integrationserfolg des Lebensbereichs Gesundheit, seiner Versorsorgungssysteme bzw. dessen bis dato unternommenen Bemühungen um Integration? Welche Handlungsbedarfe leiten sich aus der Analyse ab und wie lassen sich diese praktisch umsetzen?

\section{Migration und Gesundheit}

Die soziale und politische Integration von Migrantinnen und die Verbesserung Weg bereitender Strukturen, die einen guten Gesundheitszustand fördern, bedingen sich wechselseitig4. „Eine Gesundheitspolitik, welche die Situation der MigrantInnen einbezieht, muss deshalb Maßnahmen zur Integration und zur politischen Partizipation für Menschen mit Migrationshintergrund einschließen. ${ }^{5}$

Aufgrund der Wechseldynamik zwischen Integration und Gesundheit kann die gesundheitliche Lage Integrationsverläufe mit allen ökonomischen, sozialen, kulturellen, politischen und biologischen Implikationen abbilden. Umgekehrt spielt das Gesundheitssystem selbst eine nicht unwesentliche Rolle bei der Integration von Migranten. Migration als solche macht nicht krank oder stellt generell ein Gesundheitsrisiko dar oder: niemand wird krank nur weil er Migrant ist! ${ }^{6}$. Vielmehr birgt Migration gleichermaßen Chancen wie spezifische Belastungen für die Gesundheit. Sie bietet die Möglichkeit zur Neuorientierung und zur Nutzung alternativer ökonomischer und sozialer Ressourcen. Gelingt nach erfolgreicher Migration der Prozess der Integration hingegen nicht oder nur unzureichend, kann Migration als in erster Linie belastend, verunsichernd und somit auch krankmachend erlebt werden.

Es ist davon auszugehen, dass die Umstände der Migration sowie ethno-medizinische Hintergründe einen Einfluss auf die gesundheitliche Situation haben. Vor allem mit der Migration verbundene traumatische Erfahrungen können eine große gesundheitliche Belastung für den Betroffenen darstellen. Eine rein kulturell orientierte Perspektive, die den Blick auf den Zusammenhang Migration und Sozialstatus im aufnehmenden Land zugunsten jener, die die dazu angetan ist, die kulturelle Prägung und Nationalität zu betonen, einengt oder gar verdrängt, scheint jedoch wenig zielführend zu sein. „(...) Viel wichtiger und von gesundheitsrelevanter Bedeutung sind (...) der sozioökonomische Status, persönliche und kollektive biografische Erfahrungen sowie alters- und geschlechtsspezifische Faktoren. ${ }^{7}$

\section{Migration und sozioökonomischer Status}

Tatsache ist, dass MigrantInnen nicht selten in mehrfacher Hinsicht von sozialer Ungleichheit in Sachen Gesundheitschancen betroffen sind, wenngleich auch hier vor einer Pauschalisierung gewarnt werden muss: den/die sozial benachteiligten Migranten/in gibt es genauso wenig wie den/die sozial benachteiligten Deutsche/n!

Belegbar ist, dass eine von Migration geprägte Lebenssituation immer noch und tendenziell eher mit einem niedrigen sozioökonomischen Status, d.h. mit geringen finanziellen Ressourcen, überproportionaler Betroffenheit von Arbeitslosigkeit, mangelnder sozialer Integration, geringeren Bildungschancen etc. verbunden ist. Das biografische Ereignis Migration geht also im Vergleich zur Mehrheitsbevölkerung oft einher mit sozialer Benachteiligung im Aufnahmeland, was wiederum Auswirkungen auf die Verteilung von gesundheitlichen Chancen hat.

Die im folgenden angeführten Grundannahmen spielen beim Zugang an das Thema ,Gesundheitliche Lage von MigrantInnen' eine wesentliche Rolle:

\section{- MigrantInnen teilen die gesundheitlichen Probleme ein- heimischer PatientInnen, wenn sie sich in ihrem sozialen Status ähneln. \\ - Unterschiede im Gesund- und Kranksein korrelieren mit Variablen der Sozialisation und Herkunft ${ }^{8}$.}

\section{Migration - Integration- Gesundheitsversorgung}

Neben den in Zusammenhang mit Migration stehenden pathogenetischen Faktoren sind vor allem jene Überlegungen von Bedeutung, die danach fragen, inwiefern MigrantInnen eine adäquate kurative wie präventive Versorgung erhalten bzw. in Anspruch nehmen. Dies schließt selbstverständlich mit ein, danach zu fragen, welche Gründe ein Ausgeschlossensein verantwortet.

Die Integration von Migranten in das derzeitige präventive Regelangebot der Gesetzlichen Krankenversicherung ist - ebenso wie bei anderen Bevölkerungsgruppen in sozial ähnlichen Lagen - derzeit oft noch defizitär und lässt Wünsche in vielerlei Hinsicht bislang unerfüllt. Mit Blick auf präventive Maßnahmen sei hier exemplarisch besonders die Inanspruchnahme von Vorsorgeleistungen zu nennen. 
Trotz grundsätzlicher Zugangsoffenheit zum Angebot der GKV-Vorsorgeleistungen für alle (mit Ausnahmen, denn dies trifft u.a. nicht für AsylbewerberInnen zu) sind MigrantInnen bei der Inanspruchnahme zahnmedizinischer Präventivmaßnahmen deutlich unterrepräsentiert, während zugleich das gesundheitliche Risiko mit abnehmendem Sozialstatus steigt. Auch Vorsorgeuntersuchungen in der Schwangerschaft werden seltener wie auch später in Anspruch genommen, während die kindliche Morbidität nach der Geburt höher ist als bei deutschen Müttern?

Ein weiteres Beispiel aus dem Bereich der HIV-Prävention veranschaulicht, dass Angebotsformen nicht immer die Lebensrealität von Migrant/innen treffen: zwar liegt der Anteil der an AIDS erkrankten Migrant/innen nur geringfügig über dem der deutschen Bevölkerung, das Spektrum der Übertragungswege und die Geschlechterstruktur weichen jedoch deutlich von dem der Mehrheitsbevölkerung ab. Ursache hierfür kann sein, dass es sich meist nicht um Angehörige der seit Jahren ansässigen ausländischen Wohnbevölkerung, sondern in erster Linie um relativ neu zugereiste Migrant/innen handelt; zudem bestehen aufgrund möglicher differierender kultureller Sexualitätskonzepte und im Aufnahmeland verändernder Sexualpraktiken andere Voraussetzungen bezüglich der Übertragung von HIV und anderer sexuell übertragbarer Erkrankungen. Vor diesem Hintergrund können auf die Mehrheitsbevölkerung und ihre Gegebenheiten zugeschnittene Initiativen zur Sexualaufklärung und HIV-Prävention für diese Zielgruppe nur begrenzt wirksam sein und müssten folglich angepasst werden. ${ }^{10}$

Diese Beispiele sind Indikatoren für Hindernisse bezüglich der gesamtgesellschaftlichen Effektivität von Präventionsangeboten und zeigen, dass grundsätzlich gleiche Nutzungsrechte wie auch - möglichkeiten noch keine gleiche Nutzungseffektivität garantieren: ${ }^{11}$

So behindern informationsbedingte, kulturelle und kommunikative Barrieren die Nutzung präventiver und gesundheitsförderlicher Angebote und Möglichkeiten.

Präventionsangebote sind in vielen Fällen vornehmlich für einen bestimmten Kulturkreis und am sozialen Mittelstand orientiert konzipiert. ,Gesundheit' ist eher ein Thema der gut situierten Bevölkerung, der viele MigrantInnen wie auch andere sozial benachteiligte Gruppen häufig nicht angehören. Somit wirkt sie im Alltag dieser Bevölkerungsgruppen tendenziell nicht motivbildend. Gesundheit als erstrebenswertes Gut, nicht zu sprechen gar als ,life-style-Konzept ' „(...) wird allerdings erst dann (...) handlungsleitend, wenn die Grundbedürfnisse nach Essen, Wohnung, Sicherheit virtuell dauererfüllt sind (... $)^{12}$

Vorsorge und Prophylaxe sind in vielen Herkunftsländern nicht von herausragender Bedeutung bzw. oft nicht kulturell durch Sozialisation mit Handlungsmustern gesichert.

An der Zielgruppe „Migrant/innen” wird sichtbar, was sich bezüglich der Mechanismen und Auswirkungen auch auf andere gesellschaftliche Gruppen übertragen lässt. Bezüglich dieser Einflüsse vorhandene Potenziale zu fördern und Problemstrukturen zu reduzieren, sollte daher ein übergeordnetes Ziel migrations- (oder soll man sagen: integrations-?) sensibler Präventionsarbeit sein, denn: die -zugegebener Maßen manchmal sehr gut gemeinte- Unterstellung einer Gleichheit in Sachen Zugang $z u$ und Nutzung von Präventions- und gesundheitichen Versorgungsangeboten, ist falsch, verhindert Analyse und Verhältnisänderung und fördert damit Chancenungleichheit.

\section{Migrationserfahrungen als wertvolle Ressource}

Gleichwohl verfügen MigrantInnen sowohl im Kontext ihrer jeweiligen Prägung als auch auf Grund ihrer im Rahmen von Migrationserfahrung entwickelten Bewältigungsstrategien über spezifische gesundheitsförderliche Ressourcen. Versteht man Gesundheit als „(...)multidimensionales Kontinuum, welches auf körperlicher, psychischer und sozialer Ebene zwischen maximaler und fehlender Gesundheit variiert, so wechselt, dem salutogenetischem Ansatz folgend, der Blick von den pathogenetischen Risikofaktoren hin zu jenen, die der Entstehung von Gesundheit förderlich sind. ${ }^{13}$ Als Gesundheit beeinflussende Faktoren gelten u.a. der erfolgreiche Umgang mit Risiken und Belastungen wie auch körperliche, materielle, psychische, soziale und kulturelle Widerstandsressourcen zur Bewältigung von Spannungszuständen.

\section{"(...)Diese Ressourcen entstehen aus dem soziokulturellen} Kontext, die individuelle Sozialisation und Biografie und sind Voraussetzungen jeglichen Gesundheitshandelns. ${ }^{14}$

Durch Migrationserfahrungen können Bewältigungsstrategien erlernt werden, die den Einzelnen unter bestimmten Voraussetzungen flexibler und belastungsfähiger werden lassen können. Voraussetzung hierfür sind individuelle psychosozialen Ressourcen ( Coping-Strategien) wie auch das Vorfinden unterstützender Strukturen. „The key factors that determine a migrant' well-being include: whether one adapts well to the changes brought on my migration, whether one is living in a safe and healthy environment, and whether one can live in a productive, meaningful, and culturally integrated life" ${ }^{15}$

Wichtig in dem Zusammenhang sind beispielsweise die u.a. durch die Migration entstandenen, spezifischen Netzwerke der ethnischen Gemeinschaften mit ihren besonderen Unterstützungsformen. Auch intakte Familien können als wichtige Ressource zur Bewältigung des Akkulturationsstresses gedeutet werden, da starke Familien Belastungen tendenziell eher etwas entgegen setzen können, da die enge Bindung der Familienmitglieder untereinander und klare Rollenverteilungen im Familiensystem einen Schutzfaktor bilden. 


\section{Ressource Fremdsein}

Hinzu kommt, dass in einer von Globalisierung erfassten und somit stetig zunehmend an managing-diversityKonzepten interessierten Wanderungswelt „Fremd-Sein” an sich eine wertvolle Ressource ist oder werden kann: mindestens zwei Sprachen zu verstehen, sich in zwei oder mehreren Kulturen zurechtfinden zu können, Gemeinsamkeiten und Unterschiede zu kennen, dadurch eine Vermittlerrolle einnehmen, bedeutet eben nicht nur Belastung, sondern bietet auch Chancen, das eigene Potenzial im Rahmen gesamtgesellschaftlicher Entwicklungen zu entdecken und einbringen zu können. Leider wird dies auf beiden Seiten noch zu wenig in positiver Weise wahrgenommen und genutzt. Insbesondere im Gesundheits- und Sozialsystem gilt es in diesem Punkt zu erkennen, was in der Wirtschaft mit Blick auf den ökonomischen Nutzen als „Standortvorteil durch diversity” längst Allgemeingut ist.

\section{Empfehlungen}

Gesundheitsförderung für Menschen mit Migrationshintergrund kann und sollte neben übergeordneten Ansätzen in Politik und Lebensraumgestaltung die Ziele Integration bzw. interkulturelle Öffnung, ganzheitliche Ressourcenförderung zur Selbstbestimmung über die eigene Gesundheit und die Prävention spezifischer Risikofaktoren spezieller Risikogruppen beinhalten. Ein Schlüssel zum nachhaltigen Erfolg von Maßnahmen zur Gesundheistförderung von Migrantengruppen ist die Beteiligung der „Betroffenen”. Die problem-assoziierte Zielgruppe ist immer auch Teil der Lösung der mit ihr in Zusammenhang gebracht werdenden Probleme. Insgesamt können sich hinsichtlich dessen, was es präventiv und kurativ zu tun gilt, folgende Empfehlungen formuliert werden:

\section{- Bedarfsorientierte Angebote}

Programme und Angebote der Prävention und Gesundheitsförderung sollten sich inhaltlich wie auch organisatorisch bedarfsorientiert an den Lebenswelten der Zielgruppe ausrichten.

\section{- Verbesserung der Aufnahmefähigkeit}

Die Angebote sollten die Aufnahmefähigkeit für gesundheitsrelevante Informationen verbessern wie selbstverständlich zu gesundheitsförderlichen Lebensweisen motivierend wirken.

\section{- Entdeckung und Nutzung von Ressourcen}

Es gilt, Potenziale und spezifische Ressourcen im Sinne protektiver Faktoren und positiver Bewältigungsstrategien der Migrant/innen als Experten in eigener Sache zu erkennen, fördern und zu unterstützen. Die Adressatengruppe selbst ist so früh und umfangreich wie möglich einzubeziehen, um Angebote bedarfsorientiert und im günstigsten Fall nach der Prämisse „Hilfe zur Selbsthilfe” gestalten zu können.

\section{- Interkulturelle Regelversorgung}

Unter dem Stichwort „interkulturelle Öffnung” sollte die Auseinandersetzung mit Migrationszusammenhängen soweit wie möglich Bestandteil der Regelversorgung werden.

\section{- Interdisziplinäre Vernetzung}

Interdisziplinäre arbeitende Vernetzungsstrukturen müssen geschaffen werden und sind in Form von projektbezogenen Kooperationen auszubauen.

\section{- Förderung des politischen Diskurses}

Bezüglich der Gesundheitsförderung und des Ziels einer Verringerung sozial bedingter Ungleichheit von Gesundheitschancen gilt es, den politischen Diskurs zu fördern. Zukunfsfähig kann nur das sein, was auf politischer Ebene anerkannt und in Gesamtstrategien eingebunden ist. Integrationssensible Ansätze der Prävention und Gesundheitsförderung werden sich umso besser etablieren, je umfassender sie Teil allgemeiner Strategien sind und je weniger sie als exotische Attitüden eines idealistischen, romantisierenden, folkloristisch interessierten „Gutmenschentums" betrachtet werden können. Dies beinhaltet auch die Erkenntnis, „(...) dass unkritische Verallgemeinerungen von Bedürfnissen, Schwierigkeiten und kulturellen Inhalten die Individualität des Menschen aus dem Blickfeld geraten lassen kann,(...) es also letztlich weniger um den Einbezug einiger kultureller Gepflogenheiten in ein buntes Bild, sondern vielmehr um einen anderen Blickwinkel ${ }^{16}$ (...) geht.

\section{- Evaluation}

Bislang kann kein migrationsbezogener Ansatz der Gesundheistförderung als evidenz-basiert oder als „GoldStandard" angesehen werden ${ }^{17}$. Daher gilt es, in Konzeptionen von Modellinitiativen stets eine sinnvolle Form der Evaluation zu implementieren.

\section{- Adäquate Zugangswege}

Wichtig ist die Erschließung und Identifizierung angemessener Zugangswege zu einer Personengruppe, die im Falle der MigrantInnen in der Mehrheit noch nicht in ausreichendem Maße mit Komm-Strukturen der Prävention vertraut ist. Daher scheinen aufsuchende Angebotsstrukturen - mögliche Anknüpfungspunkte bieten hier die Communities der MigrantInnen - derzeit am meisten Erfolg versprechend.

\section{- Ermittlung von Daten}

In Deutschland liegen derzeit kaum umfangreiche und aussagekräftige, migrationssensible bzw. -spezifische Ge- 
sundheitsdaten vor. Belastbare Daten sind jedoch notwendig, um die gesundheitliche Lage der Migrant/innen einschätzen und bewerten sowie die Versorgungsstrukturen angemessen planen zu können. Insbesondere mangelt es an empirischen Untersuchungen zur psychosozialen Gesundheit von Migrant/innen. Neben der Ermittlung und dem Ausbau zielgruppenadäquater Zugangswege sind daher die objektiven, zielgruppenspezifischen Bedarfe zu ermitteln.

\section{- Information und Kommunikation}

Ein weiterer wesentlicher Punkt betrifft die Vermittlung von Informationen. Kommunikation ist schon intrakulturell nicht selten problematisch. Um so mehr steht und fällt der therapeutische Versorgungserfolg bei Patienten aus anderen Kulturkreisen mit der Möglichkeiten zur Verständigung. Hierbei geht es zum einen um die Vermittlung konkreter Inhalte (Stress, Bewegung, Erste Hilfe etc.), zum anderen um die Information über Strukturen des deutschen Gesundheitssystems und Möglichkeiten der Prävention und Gesundheitsförderung. Zur Nachhaltigkeit trägt hier bei, wenn nicht nur passiv Informationen weitergegeben werden, sondern vielmehr Wege zur eigenen Informationsbeschaffung eröffnet werden. Die Materialien müssen zugleich kulturell und inhaltlich angepasst werden, wenn sie die Zielgruppe nachhaltig erreichen sollen.

\section{- Institutionelle Interkulturalität}

Für die Optimierung und nachhaltige Veränderung des Bestehenden ist die zunehmende Gewinnung, Förderung und Beschäftigung von MigrantInnen als Fachkräfte, Mediatoren und Multiplikatoren für die Gesundheitsförderung und interkulturelle Kompetenzerweiterung bestehender Angebot und Projekte sowie die Bildung interkultureller Teams auszubauen.

\section{Integrationssensible Primärprävention}

Träger der GKV können im Alleingang keine grundsätzlichen Gesellschaftsprobleme kompensieren bzw. brach liegende Ressourcen mobilisieren. Jedoch können sie im Rahmen ihres gesetzlich vorgegebenen Spielraums durch ihr Handeln Anstöße liefern und den politischen Diskurs befördern.

Um im Themenfeld „Migration” primärpräventiv effektiv tätig zu werden, kann beispielsweise die Einbindung in ein Gesamtprogramm zur Umsetzung von § 20 SGB V Satz 1 ein wichtiger positiver Faktor sein, denn „(..) migrationsspezifische Ansätze, die auf die Beseitigung offensichtlicher Defizite in Prävention, Kuration und Rehabilitation abzielen, werden um so erfolgreicher sein, je mehr sie Teil aktueller "Trends" werden und nicht als Extralösung in der Schublade „wünschenswert aber unbezahlbar” abgelegt werden können"18.

\section{Beispiel: Die BKK - Initiative „Mehr Gesundheit für alle"}

Der Bundesverband der Betriebskrankenkassen leistet im Rahmen seiner Initiative „Mehr Gesundheit für alle”19 durch Projekte u.a. der Gesundheitsförderung mit und für Migrant/innen einen Beitrag zur Förderung interkultureller Kompetenz in Prävention und Gesundheitsförderung.

Die hieraus gewonnenen Erfahrungen sollen dabei dem BKK-System behilflich sein, seinen Versicherten mit Migrationshintergrund zu einer effektiveren, wie effizienteren Nutzung ihrer Gesundheitspotenziale und der Möglichkeiten des Gesundheitssystems zu verhelfen. Je mehr die Kassen im Gesundheitssystem vom „Payer” zum „Player" werden, desto mehr können diesbezügliche Kompetenzzuwächse Gewinn bringend für die Gestaltung von Versicherungsleistungen werden.

Um weiterer und unnötiger Dramatisierung des eigentlich normalen gesellschaftlichen Phänomens der Migration $^{20}$ keinen Vorschub zu leisten, gilt es gerade aufgrund des Themenfeldes sensibel dafür zu bleiben, dass kompensatorische Ansätze nicht von Defizitmodellen und stigmatisierenden Ansätzen sozialer Intervention geleitet werden. Woran zu erinnern man nicht müde werden sollte angesichts zumeist emotionalisierter und hochgradig interessegeleiteter Diskussionen: Interesse an erfolgreicher Integration haben nicht nur Zugewanderte. Es liegt ebenso in dem der aufnehmenden Bevölkerung. Dem entsprechend ist eine effektive und effiziente gesundheitliche Versorgung von größter Bedeutung nicht nur für eine derzeitige, zukünftige Patient/innenschaft mit Migrationshintergrund, sondern ist von zunehmendem Interesse gerade für die versorgenden Fachkräfte und nicht zuletzt die Kostenträger

\section{Grafik 1}

Projektbeispiel:

Mit Migranten für Migranten. Das Gesundheitsprojekt. Interkulturelle Gesundheit in Deutschland

Das MiMi-Gesundheitsprojekt ${ }^{21}$ wird vom Ethno-Medizinischen Zentrum e.V. im Auftrag des BKK Bundesverbandes durchgeführt. Mit Migranten für Migranten ist zugleich Motto und Konzept dieses Projektes zur interkulturellen Gesundheitsförderung und Prävention.

Durch mehrsprachige und kultursensible Programme soll Prävention und Gesundheitsförderung mit MigrantInnen für MigrantInnen nachhaltig ermöglicht werden. Engagierte MigrantInnen, die aufgrund ihrer Stellung oder ihres Ansehens Vertrauen in ihrer ethnischen Gruppe genießen, werden zu interkulturellen Gesundheitsmediatorlnnen für Prävention und Gesundheitsförderung geschult und setzen anschließend das Gelernte für Angehörige ihrer ethnischen Gruppe um². 


\section{Graphik 2: Gesundheit Hand in Hand. Ein Wegweiser für Migrantinnen und Migranten}

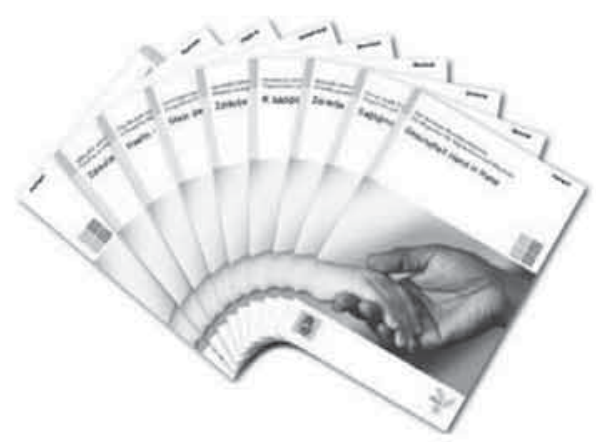

Der Gesundheitswegweiser "Gesundheit Hand in Hand - das deutsche Gesundheitssystem“ wird im Rahmen des MiMi-Projektes „Mit Migranten für Migranten" - Interkulturelle Gesundheit in Deutschland" bereits in zweiter Auflage in derzeit neun, künftig 16 Sprachen vom BKK Bundesverband und dem EthnoMedizinischen Zentrum e.V. herausgegeben. ${ }^{3}$

Inhalt des Wegweisers „Gesundheit Hand in Hand - das deutsche Gesundheitssystem

1. Die Krankenversicherung

- Wer ist gesetzlich krankenversichert?

- Freie Kassenwahl

- Leistungen der gesetzlichen Krankenkassen

- Zuzahlungen/ Eigenanteile

- Leistungen n. d. Asylbewerberleistungsgesetz

- Leistungen nach dem SGB XII (Sozialhilfe)

\section{Beim Arzt}

- Vorbereitung auf den Arztbesuch

- Gespräch mit dem Arzt

- Darüber sollte Ihnen Ihr Arzt Auskunft erteilen

- Schweigepflicht

- Regelmäßige Früherkennungsuntersuchungen

- Schutzimpfungen

\section{Beim Zahnarzt}

- Vorsorge und Bonusheft

- Zahnersatz

- Heil- und Kostenplan

- Zahnbehandlung im Ausland?

\section{Im Krankenhaus}

- Welches Krankenhaus ist das richtige für mich?

- Verordnung zur Krankenhausbehandlung (Einweisung)

- Krankenhausvertrag

- Was nehme ich mit ins Krankenhaus?

- Häufige Untersuchungen

- Aufklärung vor Operationen

- Krankenhausalltag

- Entlassung

\section{In der Apotheke}

7. Was tun im Notfall?

\section{Der öffentliche Gesundheitsdienst}

Weiterführende Adressen

Sprachen des Gesundheitswegweisers

- Deutsch • Russisch • Türkisch • Serbisch • Kroatisch • Bosnisch • Arabisch • Französisch

- Englisch

(in Vorbereitung)

- Albanisch • Persisch $\bullet$ Spanisch $\bullet$ Polnisch $\bullet$ Griechisch $\bullet$ Kurdisch-Sorani $\bullet$ Kurdisch-Kurmanci 


\section{Fußnoten}

1 Mikrozensus 2005

2 taz 07.06.06: „Jetzt amtlich: Deutschland ist Zuwanderungsland”

3 vgl. Peuckert, Rüdiger: „Integration“ in: Grundbegriffe der Soziologie, 6. Auflage, hrsg. von Bernhard Schäfers, Verlag Leske und Budrich, Opladen 2000, S. 151ff.

4 vgl. Weiss, Regula: Macht Migration krank? Eine transdisziplinäre Analyse der Gesundheit von Migrantinnen und Migranten. Seismoverlag, 2005, 2.Auflage, 10ff

5 ebd.

6 Peters, M.: Gesundheitliche und psychosoziale Versorgung von Migrantinnen und Migranten in Frankfurt. In: Gardemann, Müller, Remmers (Hrsg.): Migration und Gesundheit: Perspektiven für Gesundheitssysteme und öffentliches Gesundheitswesen, Berichte \& Materialien, Band 17. Düsseldorf: Akademie für öffentlches Gesundheitswessen in Düsseldorf, 2000: 180 - 186.

7 Weiss, Regula, 2005:ebd,

8 ebd. $282 \mathrm{f}$

9 Beauftragte der Bundesregierung für Ausländerfragen (Hrg.). Bericht der Beauftragten der Bundesregierung für Ausländerfragen übder die Lage der Ausländer in der Bundesrepublik Deutschland. Berlin/Bonn:2002/A)

10 vgl. BSFSJ (Hrg.): Familien ausländischer Herkunft in Deutschland. 6. Familienbericht. Berlin/ Bonn 2000.

11 vgl. Stickan-Verfürth: Interkulturelle Gesundheitsförderung. Die BKK, Ausgabe 04/04
12 Bauch: Das gegliederte System der gesundheitlichen Versorgung in Deutschland und Probleme der Migration. In: Türkisch Deutsche Gesundheitsstiftung (hrg.) Wissenschaftliche Arbeiten über Gesundheit und Migration. Gießen, 2002, 204ff

13 vgl. Faltermeier: Migration und Gesundheit: Fragen und Konzepte aus einer salutogenetischen und gesundheitspsychologischen Perspektive. In: Marschalck, Wiedl (Hrg.): Migration und Krankheit. IMIS-Schriften Band 10. Osnabrück: Rasch, 2001:93ff

14 ebd.

15 Weiss, Regula: ebd.

16 Stickan-Verfürth „Ältere Arbeitsmigranten in Deutschland Gesundheitliche Situation und Handlungsansätze zur Versorgungsverbesserung“, Diplom-Arbeit 2002, Uni Düsseldorf

17 ebd.

18 Schmacke: Ausgangslage und Chancen kultursensibler Prävention im Gesundheitswesen, in: Türkisch Deutsche Gesundheitsstiftung (Hrg.) Wissenschaftliche Arbeiten über Gesundheit und Migration. Gießen: Türkisch Deutsche Gesundheitsstiftung, 2202, $147 \mathrm{ff}$

19 Geene, Raimund, Philippi, Tanja (Hrsg.) Mehr Gesundheit für alle. Die BKK-Initiative als ein Modell für soziallagenbezogene Gesundheitsförderung, Wirtschaftverlag NW, 2004; siehe auch www.bkk.de/mehr-gesundheit-fuer-alle

20 vgl.Geene, Philippi 2004, ebd.

21 www.bkk-promig.de

22 ebd.

23 ebd.

\section{"Standardausstattung"}

Prof. Dr. Tobias Fröschle, www.socialnet.de

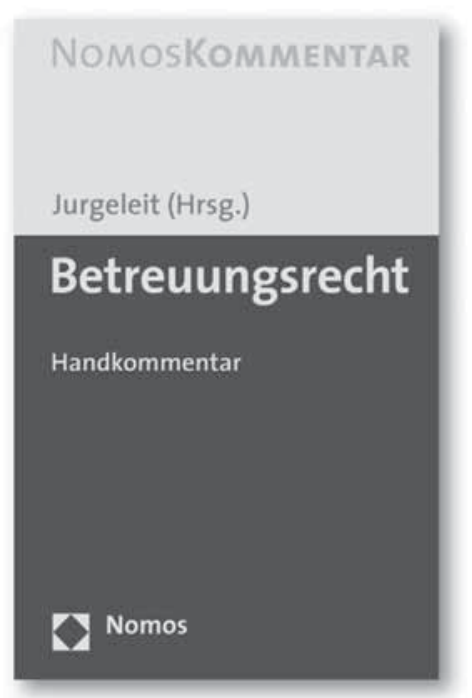

\begin{abstract}
Betreuungsrecht
Handkommentar

Herausgegeben von RiOLG Dr. Andreas Jurgeleit, Hamm

2006, 704 S., geb., 69,-€, ISBN 978-3-8329-1367-0
\end{abstract}

"Überzeugend ist bei diesem Kommentar die Praxisnähe, weshalb er sowohl für ehrenamtliche Betreuer als auch für Berufsbetreuer, Richter, Verfahrenspfleger und Betreuungsbehörden bestens geeignet ist." RA Markus Band, FGPrax 6/o6

"Handelt es sich um eine fundierte Aufbereitung der grundlegenden Vorschriften des Betreuungsrechtes, die auch für die örtlichen Betreuungsbehörden von großem Nutzen sein kann. Es ist dem Kommentar zu wünschen, dass er sich zu einer verlässlichen Hilfe für die örtliche Praxis entwickelt."

Dr. Irene Vorholz, Berlin, Der Landkreis 10/06
Bitte bestellen Sie bei Ihrer Buchhandlung oder bei Nomos 607221/2104-37| $\square-43$ | www.nomos.de | sabine.horn@nomos.de 